\title{
Un modèle du comportement dynamique d'engrenages droits avec prise en compte de contacts hors du plan d'action
}

\author{
Philippe Velex ${ }^{1, a}$, Olivier Duverger ${ }^{2}$ et Philippe Sainsot ${ }^{1}$ \\ 1 Laboratoire de Mécanique des Contacts et des Solides, INSA Lyon, UMR CNRS 5514, 69621 Villeurbanne, France \\ 2 Pôle Machines et Commandes, CETIM Senlis, 60304 Senlis, France
}

Reçu le 2 octobre 2003, accepté le 3 septembre 2004

\begin{abstract}
Résumé - Sous l'effet des charges transmises, les éléments d'une transmission par engrenages se déforment et les contacts entre dentures peuvent s'effectuer hors du plan d'action théorique entre profils non conjugués si les corrections de forme sur les dents ne sont pas appropriées. L'objectif principal de cet article est de présenter un modèle original permettant une première approche du comportement dynamique d'engrenages cylindriques droits soumis à ces conditions de fonctionnement particulières. Le pignon et la roue sont assimilés à des cylindres indéformables liés par des rigidités dont les valeurs et les orientations dans l'espace dépendent du temps afin de simuler les évolutions des conditions d'engrènement. En première approximation, seuls les degrés de liberté de torsion sont pris en considération. Les résultats montrent que les contacts entre profils non conjugués induisent des phénomènes dynamiques particuliers qui se traduisent au niveau du chargement sur les dentures par, i) une durée de contact plus importante donc un rapport de conduite plus élevé que dans les conditions nominales, ii) des impacts dont les amplitudes dépendent du niveau de charge transmise et des vitesses de rotation.
\end{abstract}

Mots clés : Engrenages / modélisation / dynamique / impacts / profils non conjugués / engagement

\begin{abstract}
A model of the dynamic behaviour of spur gears with consideration of off-line of action contacts. Because of deflections under load, contacts on tooth flanks can occur outside the theoretical plane of action between non-conjugated profiles especially when tooth modifications are not appropriate. The main objective of the present paper is to introduce an original model of the dynamic behaviour of spur gears when submitted to these particular meshing conditions. The pinion and the gear of a pair are modelled as two rigid cylinders linked by stiffnesses and dampers whose amplitudes and orientations in space vary with time in order to simulate the evolutions of the meshing conditions. For the sake of simplicity, only the two torsional degrees-of-freedom of the pinion and the gear are considered. The results show that the contacts between non-conjugated flanks lead to a particular dynamic behaviour characterised by i) an actual contact ratio larger than the theoretical one, ii) impacts at engagement whose amplitudes depend on transmitted loads and speeds.
\end{abstract}

Key words: Gears / modelling / dynamics / impacts / off line-of-action contacts / engagement

\section{Introduction}

La prédiction et le contrôle du comportement dynamique de transmissions par engrenages sont des points sensibles en termes de fiabilité et de bruit qui concernent de nombreux secteurs industriels tels que l'automobile, l'aéronautique, la production d'énergie...

Le développement des capacités de calcul a conduit à une évolution importante des modélisations mises en œuvre depuis les modèles torsionnels initiaux à un seul

a Auteur correspondant : Philippe.Velex@insa-lyon.fr degré de liberté $[1,2], \ldots$ jusqu'aux approches de type éléments finis visant à intégrer les engrenages, les arbres, les paliers, les carters dans un même modèle $[3-5], \ldots$ À un niveau plus local, les modélisations des conditions de contact instantanées ont également évolué et permettent de considérer les variations de raideur d'engrènement consécutives aux variations de longueur de contact au cours du mouvement, d'introduire certains écarts de forme (corrections, erreurs de pas, ...) et des défauts de montage des mobiles (désalignements) [6-8]. Toutefois, la très large majorité des travaux de simulation considère que les contacts sous charge sont au voisinage 
des contacts dans les conditions de corps rigides et néglige ainsi l'influence des déplacements élastiques sur les positions des engagements et dégagements réels. Ce problème n'a reçu que peu d'attention au cours des dernières décennies [9-11] et les résultats obtenus semblent relativement contradictoires.

Dans cet article, nous proposons un modèle original de comportement dynamique d'engrenages droits qui intègre les conséquences de conditions de contact particulières engendrées par des engagements prématurés hors du plan d'action théorique. Les positions des premiers points de contact pour des pignons et des roues déformés sont recherchées de manière numérique en tenant compte de la géométrie réelles des dents. Les excitations supplémentaires qui en découlent sont traduites sous forme d'impulsions qui s'annulent lors du retour à des conditions de contact entre profils conjugués. Les exemples d'application mettent en évidence la présence d'impacts et des modifications sensibles des caractéristiques géométriques de l'engrènement en fonction du niveau de charge transmis et des vitesses de rotation.

\section{Modélisation}

\subsection{Hypothèses principales - Simplifications}

Dans le cadre de cette première approche des phénomènes d'engagements hors du plan d'action, les simulations sont conduites à partir d'un modèle purement torsionnel à paramètres concentrés et seuls les degrés de liberté en torsion du pignon et de la roue sont considérés. Ces angles de torsion au voisinage des rotations de corps rigides sont supposés petits devant l'unité. L'étude se limite aux seuls engrenages droits et les dentures ne sont pas corrigées selon le profil et la largeur. Les vitesses de rotation dans les conditions de corps rigides ainsi que les couples appliqués sont constants. Enfin, les développements et les résultats sont limités aux engagements prématurés tandis que les phénomènes de même nature se produisant au moment du dégagement ne sont pas abordés dans cet article.

\subsection{Position des points de contact et différence de vitesse normale à l'engagement}

Les charges transmises dans une transmission par engrenages déforment les différents éléments et peuvent conduire pour des dentures non corrigées ou insuffisamment corrigées à des contacts hors du plan d'action entre profils non conjugués. La détermination des positions des points de contact pour ces engagements prématurés est un problème géométrique complexe qui a été abordé ici de manière purement numérique selon le schéma suivant:

a) les profils de 3 paires de dents successives sur le pignon et la roue sont discrétisés et les positions des points de contact théoriques (pour des corps indéformables) et leur évolution au cours du mouvement sont déterminées ;

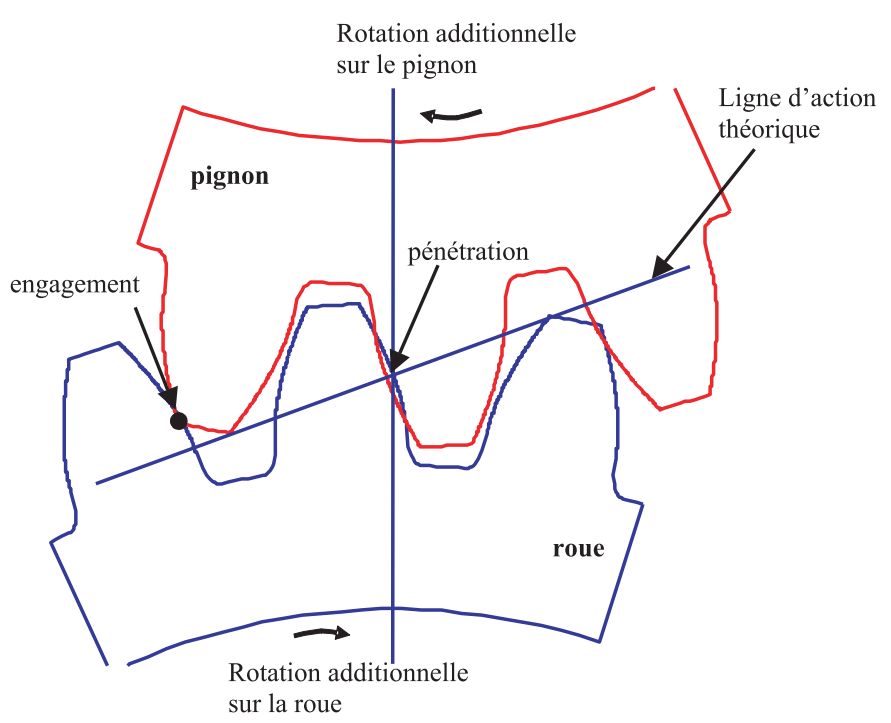

Fig. 1. Schéma de principe pour la recherche du premier point de contact.

b) en accord avec les hypothèses d'un modèle purement torsionnel, deux rotations respectivement sur le pignon et la roue sont superposées afin de simuler les déflexions élastiques, l'interpénétration des deux corps correspond alors à la déflexion au contact (Fig. 1) ;

c) la position du premier point de contact est ensuite calculée de manière incrémentale et correspond à l'état du système pour lequel la distance entre le sommet de dent du pignon et le profil en vis-à-vis de la dent de la roue est minimale.

Les profils n'étant plus conjugués lors d'un engagement prématuré, leurs vitesses dans la direction normale au contact sont différentes. En considérant que la normale commune $\vec{n}_{M}^{0}$ à l'engagement (point $M^{0}$ ) est la normale au profil menant (ce qui est théoriquement impose un léger arrondi en sommet des dents de la roue), il vient, dans les conditions de corps rigides, la composante de vitesse supplémentaire :

$$
\begin{aligned}
\Delta V^{0} & =\omega_{1}\left(O_{1} \vec{M}_{1} \wedge \vec{n}_{M}^{0}\right) \cdot \vec{z}-\omega_{2}\left(O_{2} \vec{M}_{2} \wedge \vec{n}_{M}^{0}\right) \cdot \vec{z} \\
& =\lambda_{M}^{0} \omega_{1}
\end{aligned}
$$

avec $M_{1}, M_{2}$ : deux point appartenant respectivement au pignon et à la roue en contact au point $M_{0}$

$$
\lambda_{M}^{0}=\gamma_{0}\left(R b_{1}+R b_{2}\right) \frac{R b_{1}}{R b_{2}} \operatorname{tg} \alpha_{\mathrm{p}}
$$

$\omega_{1}$ : vitesse de rotation du pignon dans les conditions de corps rigides, $R b_{1}, R b_{2}$ : rayons de base du pignon et de la roue, $\alpha_{\mathrm{p}}$ : angle de pression de fonctionnement, $\gamma_{0}$ représente l'angle de pression additionnel définissant partiellement la position du premier point de contact (Fig. 2).

En supposant que $\gamma_{0}$ est faible devant l'unité de sorte que $\cos \gamma_{0} \cong 1$ et $\sin \gamma_{0} \cong \gamma_{0}$, la fermeture de 


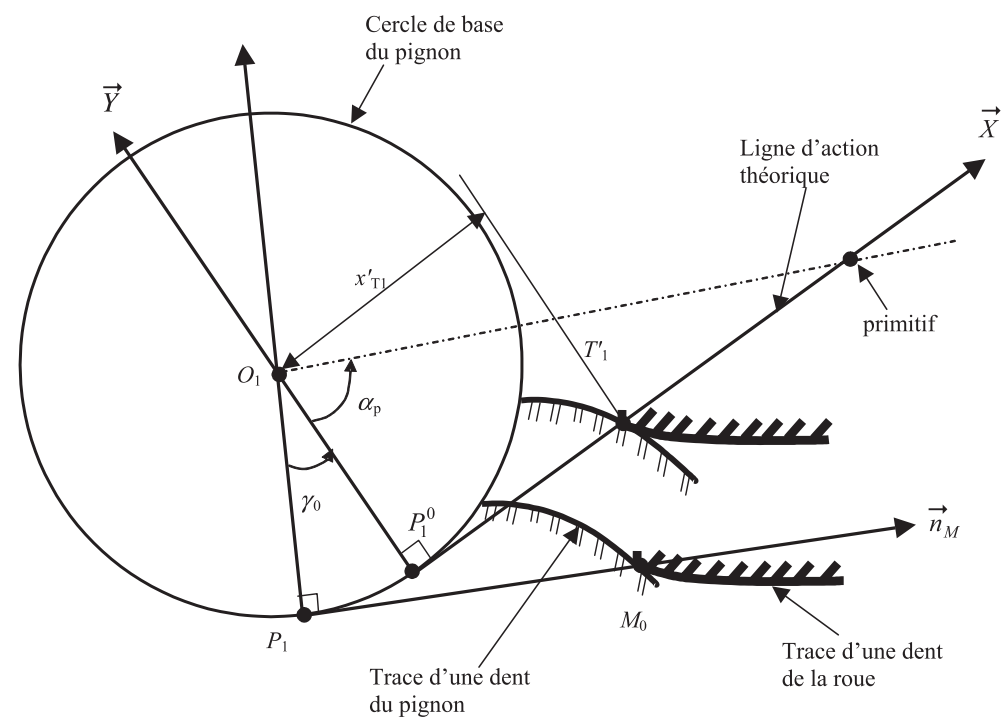

Fig. 2. Paramètres géométriques à l'engagement hors du plan d'action.

Tableau 1. Données engrenages.

\begin{tabular}{lc}
\hline Nombre de dents & 25 \\
Module $(\mathrm{mm})$ & 4 \\
$\alpha_{\mathrm{p}}\left({ }^{\circ}\right)$ & 20 \\
Déports $(\mathrm{mm})$ & 0 \\
Largeurs $(\mathrm{mm})$ & 25 \\
\hline
\end{tabular}

chaîne vectorielle dans le polygone $\left(O_{1}, P_{1}^{0}, T_{1}^{\prime}, M^{0}, P_{1}\right)$ conduit à :

$$
\begin{aligned}
\gamma_{0} & \cong-\frac{x_{T 1}^{\prime}}{m Z_{1} \cos \alpha_{\mathrm{p}}}\left[1+\left(\frac{\Delta x}{x_{T 1}^{\prime}}\right)\right. \\
& \left.-\sqrt{1-2\left(\frac{\Delta x}{x_{T 1}^{\prime}}\right)-2 Z_{1} \cos \alpha_{\mathrm{p}}\left(\frac{\Delta y}{x_{T 1}^{\prime}}\right)\left(\frac{m}{x_{T 1}^{\prime}}\right)}\right]
\end{aligned}
$$

avec

$$
\begin{aligned}
& x_{T 1}^{\prime}=P_{1}^{0} T_{1}^{\prime}=\frac{m}{2}\left[\sin \alpha_{\mathrm{p}}\left(Z_{1}+Z_{2}\right)\right. \\
& \left.-Z_{2} \sqrt{\left(1+\frac{2 h a_{2}}{Z_{2}}\right)^{2}-\cos ^{2} \alpha_{\mathrm{p}}}\right],
\end{aligned}
$$

position du premier point de contact entre corps rigides, $\Delta x, \Delta y$ : écarts de position selon $\vec{X}$ et $\vec{Y}$ entre les premiers points de contact réel et théorique, $m$ : module, $Z_{1}, Z_{2}$ : nombre de dents sur le pignon, sur la roue, $h a_{2}$ : coefficient de saillie sur la roue.

Les applications numériques qui suivent ont été conduites pour un pignon et une roue identiques dont les caractéristiques dimensionnelles principales sont portées dans le tableau 1.

Les résultats en termes de position du premier point de contact et de différence de vitesse normale à l'engagement
Tableau 2. Résultats sur la position du point d'engagement et la différence de vitesse normale.

\begin{tabular}{cccc}
\hline $\begin{array}{c}\text { Interpénétration } \\
(\mu \mathrm{m})\end{array}$ & $\begin{array}{c}\Delta x \\
(\mathrm{~mm})\end{array}$ & $\begin{array}{c}\Delta y \\
(\mathrm{~mm})\end{array}$ & $\begin{array}{c}\lambda_{M}^{0} \\
(\mathrm{~mm})\end{array}$ \\
\hline 0 & 0 & 0 & 0 \\
10 & $-0,5643$ & $-0,3238$ & 1,25 \\
40 & $-1,1813$ & $-0,689$ & 2,4 \\
100 & $-1,838$ & $-1,09$ & 3,48 \\
\hline
\end{tabular}

sont donnés dans le tableau 2 pour différentes valeurs de pénétration ou d'écrasement au niveau du couple de dents en prise juste avant l'engagement hors du plan d'action.

\subsection{Modèle dynamique}

De manière classique, l'état de référence (avant déformation) correspond aux mouvements de corps rigides. D'après la figure 3 , la déflexion au point de contact courant $M$ peut s'exprimer, dans le cadre des petits déplacements, par :

$$
\Delta(M)=\left[\vec{u}_{1}^{R}\left(M_{1}\right)-\vec{u}_{2}^{R}\left(M_{2}\right)\right] \cdot \vec{n}_{M}-M_{1}^{0} \vec{M}_{2}^{0} \cdot \vec{n}_{M}
$$

avec $\vec{u}_{1}^{R}\left(M_{1}\right), \vec{u}_{2}^{R}\left(M_{2}\right)$, les vecteurs déplacements au point $M_{1}$ (lié au pignon) et $M_{2}$ (lié à la roue), $\vec{n}_{M}$, la normale commune au point de contact (extérieure au flanc menant par convention), $M_{1}^{0}, M_{2}^{0}$, positions initiales des points entrés en contact en $M$ à l'état déformé.

En considérant le modèle torsionnel simple caractérisé par deux angles infinitésimaux $\theta_{1}$ et $\theta_{2}$ pour le pignon et la roue respectivement (Fig. 4), le formalisme des torseurs de petits déplacements conduit à :

$\Delta(M)=\left(O_{1} \vec{M}_{1} \wedge \vec{n}_{M}\right) \cdot \vec{z} \theta_{1}-\left(O_{2} \vec{M}_{2} \wedge \vec{n}_{M}\right) \cdot \vec{z} \theta_{2}-\delta_{M}(\tau)$ 


$$
\begin{aligned}
& {\left[\begin{array}{cc}
J_{1} & 0 \\
0 & J_{2}
\end{array}\right]\left[\begin{array}{l}
\ddot{\theta}_{1} \\
\ddot{\theta}_{2}
\end{array}\right]+\left[\begin{array}{cc}
\int_{M} \rho_{1 M}^{2}(\tau) c(M) \mathrm{d} M & -\int_{M} \rho_{1 M}(\tau) \cdot \rho_{2 M}(\tau) c(M) \mathrm{d} M \\
-\int_{M}^{M} \rho_{1 M}(\tau) \cdot \rho_{2 M}(\tau) c(M) \mathrm{d} M & \int_{M} \rho_{2 M}^{2}(\tau) c(M) \mathrm{d} M
\end{array}\right]\left[\begin{array}{c}
\dot{\theta}_{1} \\
\dot{\theta}_{2}
\end{array}\right]} \\
& +\left[\begin{array}{cc}
\int_{M} \rho_{1 M}^{2}(\tau) k(M) \mathrm{d} M & -\int_{M} \rho_{1 M}(\tau) \cdot \rho_{2 M}(\tau) k(M) \mathrm{d} M \\
-\int_{M}^{\rho_{1 M}(\tau) \cdot \rho_{2 M}(\tau) k(M) \mathrm{d} M} & \int_{M} \rho_{2 M}^{2}(\tau) k(M) \mathrm{d} M
\end{array}\right]\left[\begin{array}{l}
\theta_{1} \\
\theta_{2}
\end{array}\right]=\left[\begin{array}{l}
C_{1} \\
C_{2}
\end{array}\right] \\
& +\left[\begin{array}{c}
\int_{M} \rho_{1 M}(\tau) \cdot k(M) \cdot \delta_{M}(\tau) \cdot \mathrm{d} M \\
-\int_{M} \rho_{2 M}(\tau) \cdot k(M) \cdot \delta_{M}(\tau) \cdot \mathrm{d} M
\end{array}\right]+\omega_{1}\left[\begin{array}{c}
\int_{M} \rho_{1 M}(\tau) \cdot \lambda_{M}(\tau) \cdot c(M) \cdot \mathrm{d} M \\
-\int_{M} \rho_{2 M}(\tau) \cdot \lambda_{M}(\tau) \cdot c(M) \cdot \mathrm{d} M
\end{array}\right]
\end{aligned}
$$

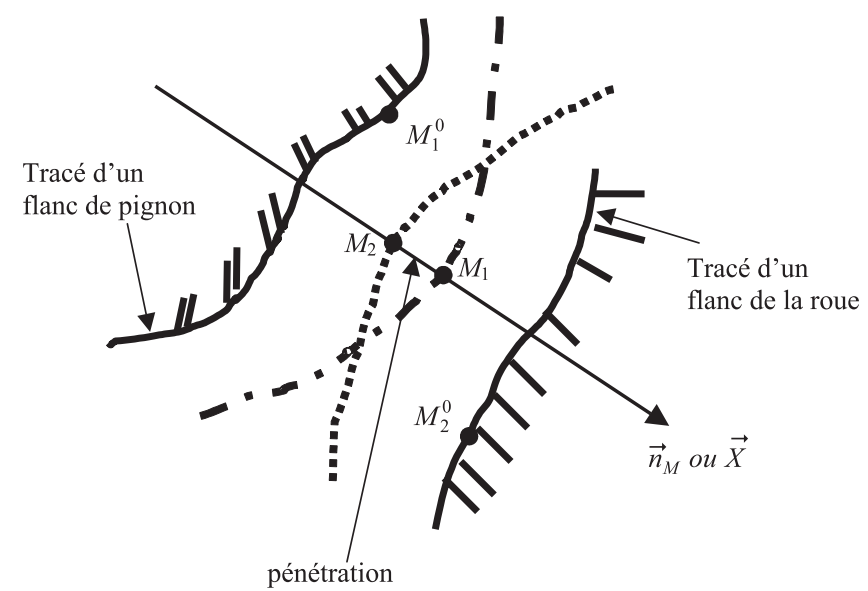

Fig. 3. Conditions de contact (traits continus : positions avant déformation, traits en pointillés : positions à l'état déformé).

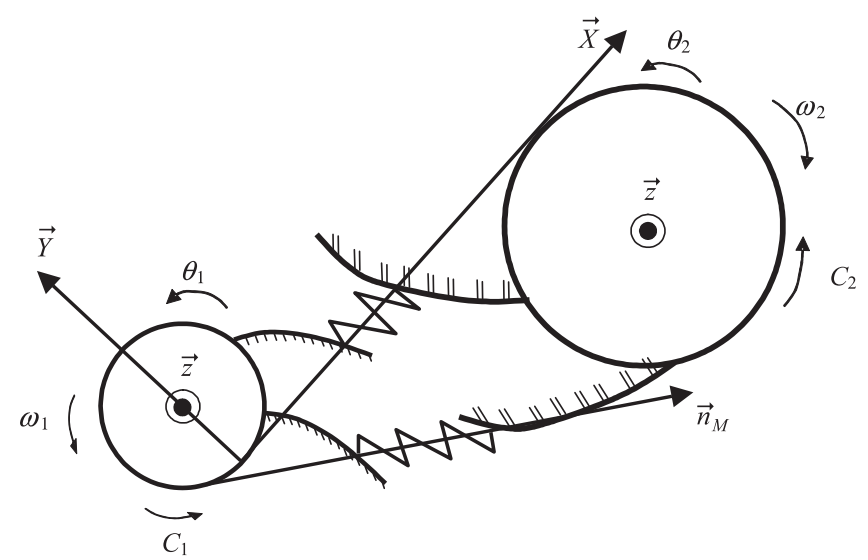

Fig. 4. Modèle dynamique (par souci de clarté, les amortissements ne sont pas représentés).

où $\tau$ est le temps adimensionné par rapport à la période d'engrènement et $\delta_{M}(\tau)=M_{1}^{0} \vec{M}_{2}^{0} \cdot \vec{n}_{M}$ est la séparation normale initiale entre les points en contact.
Il est important de signaler que, pour des dentures sans correction, $\delta_{M}(\tau)$ est différent de zéro uniquement lorsque les contacts s'effectuent hors du plan d'action. La normale $\vec{n}_{M}$ évolue avec le temps et tend vers $\vec{X}$ lorsque le contact retourne sur le plan d'action. En introduisant pour la paire de dents en contact $\mathrm{n}^{\circ} i$, la fonction $\phi_{i}(\tau)$ définie schématiquement sur la figure 5 , on peut écrire :

$$
\vec{n}_{M} \cong \vec{X}-\gamma_{0} \phi_{i}(\tau) \vec{Y}
$$

et la déflexion au point $M$ s'exprime finalement

$$
\Delta(M)=\rho_{1 M}(\tau) \theta_{1}-\rho_{2 M}(\tau) \theta_{2}-\delta_{M}(\tau)
$$

avec $\rho_{1 M}(\tau)=R b_{1}$

$$
\rho_{2 M}(\tau)=-R b_{2}+\gamma_{0} \phi_{i}(\tau)\left(R b_{1}+R b_{2}\right) \operatorname{tg} \alpha_{\mathrm{p}}
$$

De manière analogue, la différence de vitesse normale totale (incluant les vitesses de corps rigides et les dérivées des degrés de liberté $\theta_{1}$ et $\theta_{2}$ ) s'écrit après simplification par la condition cinématique $R b_{1} \omega_{1}+R b_{2} \omega_{2}=0$ :

$$
\Delta V(M)=\rho_{1 M}(\tau) \dot{\theta}_{1}-\rho_{2 M}(\tau) \dot{\theta}_{2}+\lambda_{M}(\tau) \omega_{1}
$$

$\operatorname{avec} \lambda_{M}(\tau)=\lambda_{M}^{0} \phi_{i}(\tau)$.

Les contacts entre les dents du pignon et de la roue sont supposés linéiques et l'effort au point de contact $M$ est déduit de la loi de comportement linéaire :

$$
\mathrm{d} \vec{F}_{1 \rightarrow 2}(M)=[k(M) \Delta(M)+c(M) \Delta V(M)] \mathrm{d} M \vec{n}_{M}
$$

où $k(M)$ et $c(M)$ sont respectivement les raideurs et amortissements par unité de longueur au point $M$.

Le théorème du moment dynamique conduit alors au système différentiel :

$$
\text { voir équation (9) ci-dessus }
$$

avec $J_{1}, J_{2}$ : les moments d'inertie polaire du pignon et de la roue, $C_{1}, C_{2}$ : les couples appliqués sur le pignon et la roue.

En ne gardant que les termes du premier ordre et en considérant que, pour des dentures droites, il n'y a pas de 


$$
\begin{aligned}
& {\left[\begin{array}{ll}
J_{1} & 0 \\
0 & J_{2}
\end{array}\right]\left[\begin{array}{l}
\ddot{\theta}_{1} \\
\ddot{\theta}_{2}
\end{array}\right]+C(\tau)\left[\begin{array}{cc}
R b_{1}^{2} & R b_{1} R b_{2} \\
R b_{1} R b_{2} & R b_{2}^{2}
\end{array}\right]\left[\begin{array}{c}
\dot{\theta}_{1} \\
\dot{\theta}_{2}
\end{array}\right]+K(\tau)\left[\begin{array}{cc}
R b_{1}^{2} & R b_{1} R b_{2} \\
R b_{1} R b_{2} & R b_{2}^{2}
\end{array}\right]\left[\begin{array}{l}
\theta_{1} \\
\theta_{2}
\end{array}\right]} \\
& -\left(R b_{1}+R b_{2}\right) \operatorname{tg} \alpha_{\mathrm{p}} b\left[\begin{array}{cc}
0 & R b_{1} \sum_{i} \gamma_{0} \phi_{i}(\tau) c_{i}(\tau) \\
R b_{1} \sum_{i} \gamma_{0} \phi_{i}(\tau) c_{i}(\tau) & 2 R b_{2} \sum_{i} \gamma_{0} \phi_{i}(\tau) c_{i}(\tau)
\end{array}\right]\left[\begin{array}{l}
\dot{\theta}_{1} \\
\dot{\theta}_{2}
\end{array}\right] \\
& -\left(R b_{1}+R b_{2}\right) \operatorname{tg} \alpha_{\mathrm{p}} b\left[\begin{array}{cc}
0 & R b_{1} \sum_{i} \gamma_{0} \phi_{i}(\tau) k_{i}(\tau) \\
R b_{1} \sum_{i} \gamma_{0} \phi_{i}(\tau) k_{i}(\tau) & 2 R b_{2} \sum_{i} \gamma_{0} \phi_{i}(\tau) k_{i}(\tau)
\end{array}\right]\left[\begin{array}{l}
\theta_{1} \\
\theta_{2}
\end{array}\right] \\
& =\left[\begin{array}{l}
C_{1} \\
C_{2}
\end{array}\right]+b \sum_{i} k_{i}(\tau) \delta_{i}(\tau)\left[\begin{array}{l}
R b_{1} \\
R b_{2}
\end{array}\right]-b \omega_{1} R b_{1}\left(R b_{1}+R b_{2}\right) \operatorname{tg} \alpha_{\mathrm{p}}\left[\begin{array}{c}
\sum_{i}\left\{\frac{R b_{1}}{R b_{2}} \gamma_{0} \phi_{i}(\tau)\right\} c_{i}(\tau) \\
\sum_{i}\left\{\gamma_{0} \phi_{i}(\tau)\right\} c_{i}(\tau)
\end{array}\right] \\
& -\left(R b_{1}+R b_{2}\right) \operatorname{tg} \alpha_{\mathrm{p}} b\left[\begin{array}{c}
0 \\
\sum_{i} \gamma_{0} \phi_{i}(\tau) \delta_{i}(\tau) k_{i}(\tau)
\end{array}\right]
\end{aligned}
$$

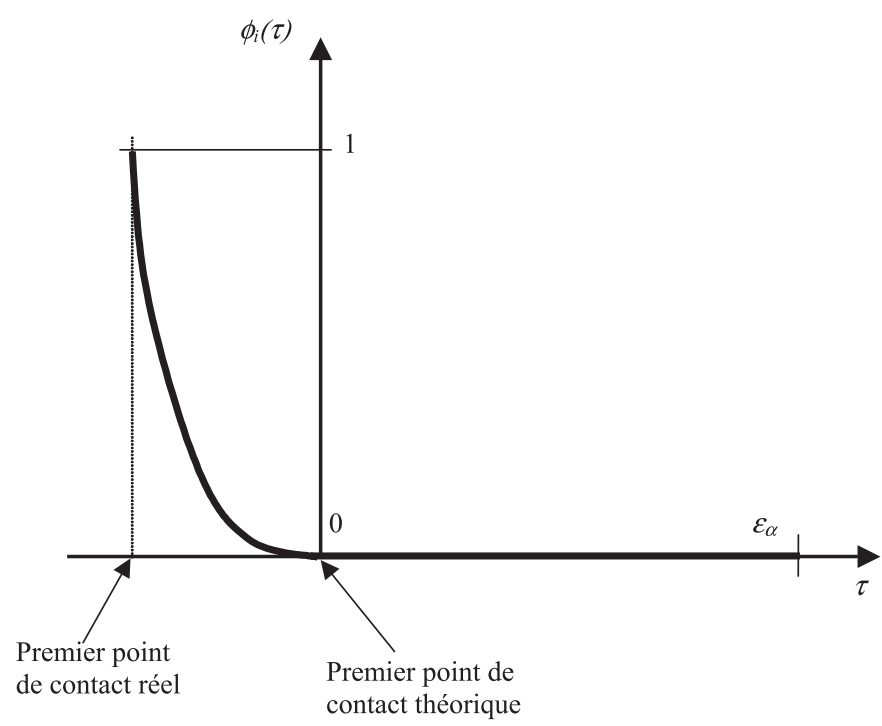

Fig. 5. Fonction $\phi_{i}(\tau)$ ( $\varepsilon_{\alpha}$ représente le rapport de conduite).

variation de géométrie et de loi de comportement selon la largeur des dents, l'équation (9) peut se réécrire sous forme discrète comme :

$$
\text { voir équation (10) ci-dessus }
$$

où l'indice $i$ se rapporte à la paire de dents en prise $\mathrm{n}^{\circ} i$, $C(\tau)=\sum_{i} c_{i}(\tau)$ et $K(\tau)=\sum_{i} k_{i}(\tau)$.

Supposant de plus, que les raideurs et amortissements pour une seule paire de dents sont sensiblement constants au cours du cycle d'engrènement, c'est-à-dire, $b c_{i}(\tau) \cong c_{I}$ et $b k_{i}(\tau) \cong k_{I}$ et que les variations sur les différents paramètres de position entre l'engagement prématuré et le retour sur le plan d'action obéissent à des lois similaires, soit, $\delta_{i}(\tau)=\delta_{0} \phi_{i}(\tau)$, on obtient finalement :

$$
\begin{gathered}
{\left[\begin{array}{ll}
J_{1} & 0 \\
0 & J_{2}
\end{array}\right]\left[\begin{array}{l}
\ddot{\theta}_{1} \\
\ddot{\theta}_{2}
\end{array}\right]+C(\tau)\left[\begin{array}{cc}
R b_{1}^{2} & R b_{1} R b_{2} \\
R b_{1} R b_{2} & R b_{2}^{2}
\end{array}\right]\left[\begin{array}{l}
\dot{\theta}_{1} \\
\dot{\theta}_{2}
\end{array}\right]} \\
+K(\tau)\left[\begin{array}{cc}
R b_{1}^{2} & R b_{1} R b_{2} \\
R b_{1} R b_{2} & R b_{2}^{2}
\end{array}\right]\left[\begin{array}{l}
\theta_{1} \\
\theta_{2}
\end{array}\right] \\
-\left(R b_{1}+R b_{2}\right) \operatorname{tg} \alpha_{\mathrm{p}} c_{I} \gamma_{0} \sum_{i} \phi_{i}(\tau)\left[\begin{array}{cc}
0 & R b_{1} \\
R b_{1} & 2 R b_{2}
\end{array}\right]\left[\begin{array}{l}
\dot{\theta}_{1} \\
\dot{\theta}_{2}
\end{array}\right] \\
-\left(R b_{1}+R b_{2}\right) \operatorname{tg} \alpha_{\mathrm{p}} k_{I} \gamma_{0} \sum_{i} \phi_{i}(\tau)\left[\begin{array}{c}
0 \\
R b_{1} \\
R b_{1} 2 R b_{2}
\end{array}\right]\left[\begin{array}{l}
\theta_{1} \\
\theta_{2}
\end{array}\right] \\
=\left[\begin{array}{l}
C_{1} \\
C_{2}
\end{array}\right]+k_{I} \delta_{0} \sum_{i} \phi_{i}(\tau)\left[\begin{array}{l}
R b_{1} \\
R b_{2}
\end{array}\right] \\
-\left(R b_{1}+R b_{2}\right) \operatorname{tg} \alpha_{\mathrm{p}} k_{I} \delta_{0} \gamma_{0} \sum_{i} \phi_{i}(\tau) \\
-c_{I} \omega_{1} R b_{1}\left(R b_{1}+R b_{2}\right) \operatorname{tg} \alpha_{\mathrm{p}} \gamma_{0} \sum_{i} \phi_{i}(\tau)\left[\begin{array}{l}
R b_{1} / R b_{2} \\
1
\end{array}\right]
\end{gathered}
$$




\section{Résultats}

Pour les applications numériques qui suivent, les fonctions impulsions $\phi_{i}(\tau)$ sont approchées par deux segments de droites passant par les trois points caractéristiques (premier point de contact réel, premier point de contact théorique et dégagement en $\tau=\varepsilon_{\alpha}$ ). Les équations du mouvement sont résolues par un schéma temporel implicite à pas de temps variable [12]. Les résultats portent sur les efforts instantanés sur une paire de dents en contact pour trois vitesses différentes (1500, 4000 et 15000 t. $\mathrm{min}^{-1}$ ) et les trois conditions suivantes :

a- en négligeant les engagements hors du plan d'action théorique;

b- avec engagements prématurés et charge faible;

c- avec engagements prématurés et charge élevée.

Les efforts de contact adimensionnés par rapport à l'effort statique maximal (paramètre $R$ ) sont représentés en fonction du temps adimensionné $\tau$ pour différentes valeurs de charge (couples de 50 et $200 \mathrm{Nm}$ ) et deux valeurs de facteur d'amortissement (2 et $5 \%$ de l'amortissement critique) (Figs. 6 et 7).

Les principales observations qui peuvent en être déduites sont listées ci-dessous :

a- En accord avec les résultats de Vedmar et Henriksson [11], les engagements hors du plan d'action induisent une augmentation du rapport de conduite réel, c'est-à-dire, une durée de contact plus importante pour une dent. De manière logique, cette durée de contact augmente avec la charge transmise puisqu'à mesure que les déflexions augmentent, les engagements se font de plus en plus tôt.

b- En parallèle, les engagements prématurés (hors du plan d'action théorique) sont susceptibles d'entraîner des impacts (surcharges) lors de la prise de contact. Les amplifications d'efforts (par rapport au chargement statique) apparaissent principalement aux très grandes vitesses et sont plus élevées pour des dentures faiblement chargées que pour les dentures fortement chargées.

c- À l'extérieur de la zone d'engagement, les diagrammes d'effort sont relativement proches de ceux obtenus en négligeant les engagements hors du plan d'action. Cette observation souligne le caractère localisé des conséquences d'engagements prématurés.

d- Le modèle d'amortissement utilisé apparaît comme un paramètre sensible puisqu'une partie non négligeable de la surcharge à l'engagement ainsi que la position du maximum d'effort dépendent de la composante visqueuse dans l'expression de l'effort de contact (8). On remarque, en particulier, que les surcharges à l'engagement croissent avec un amortissement plus élevé. Compte tenu des conditions particulières lors d'engagements entre profils non conjugués (influence des effets d'écrasement du film lubrifiant en particulier), ce point est certainement critique et nécessite des analyses supplémentaires.
$\mathrm{R}$

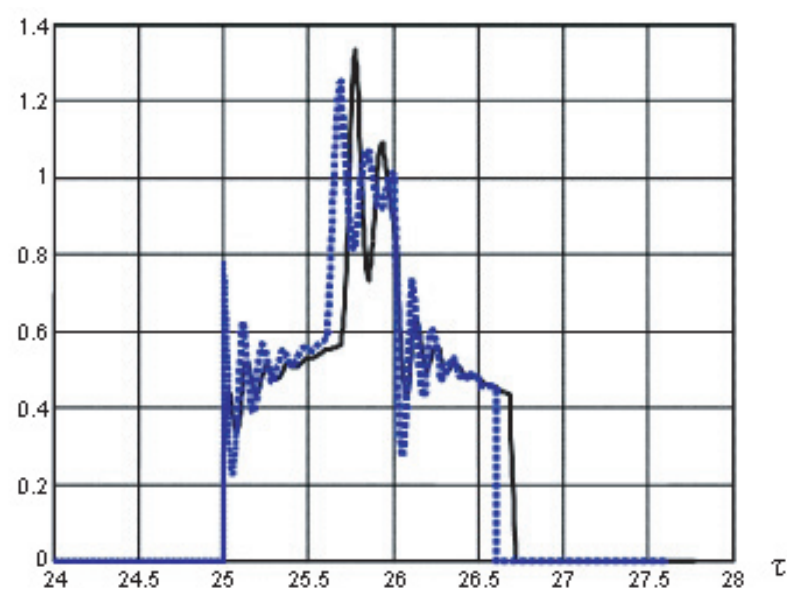

(a)

$\mathrm{R}$

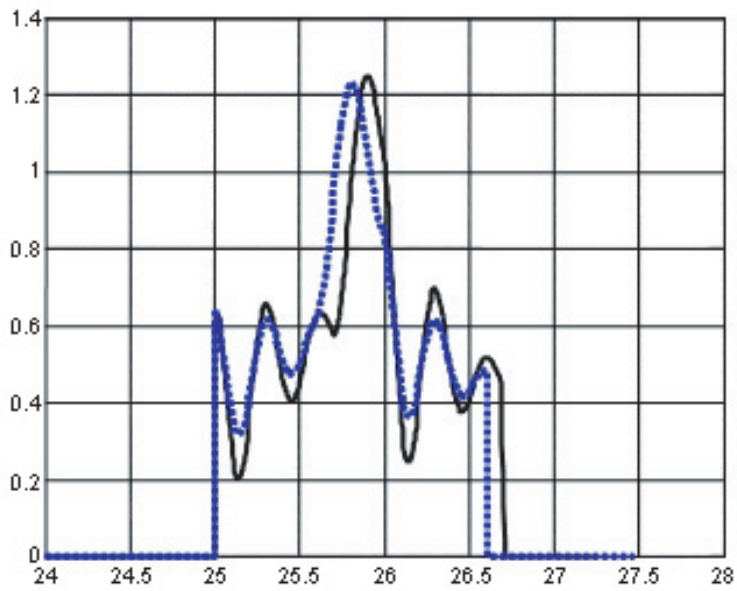

(b)

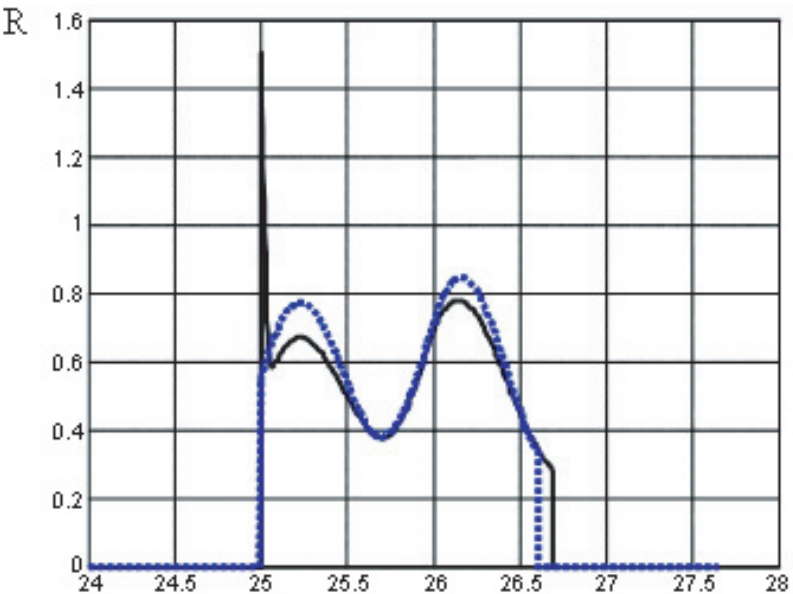

(c)

Fig. 6. Efforts sur une paire de dents en prise à différentes vitesses (facteur d'amortissement de $5 \%$ - couple de $50 \mathrm{Nm}$ ) (— Engagements prématurés, ...... Référence), (a) vitesse nominale de 1500 t.min ${ }^{-1}$, (b) vitesse nominale de 4000 t.min ${ }^{-1}$, (c) vitesse nominale de 15000 t.min ${ }^{-1}$. 
$\mathrm{R}$

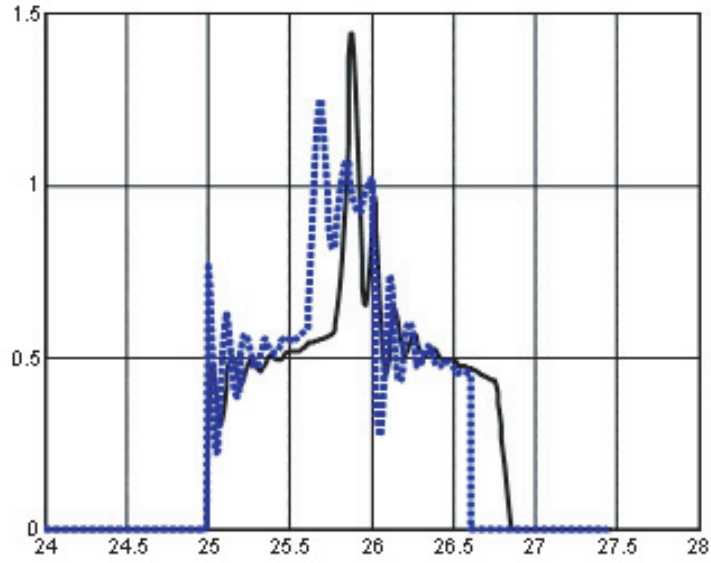

(a)

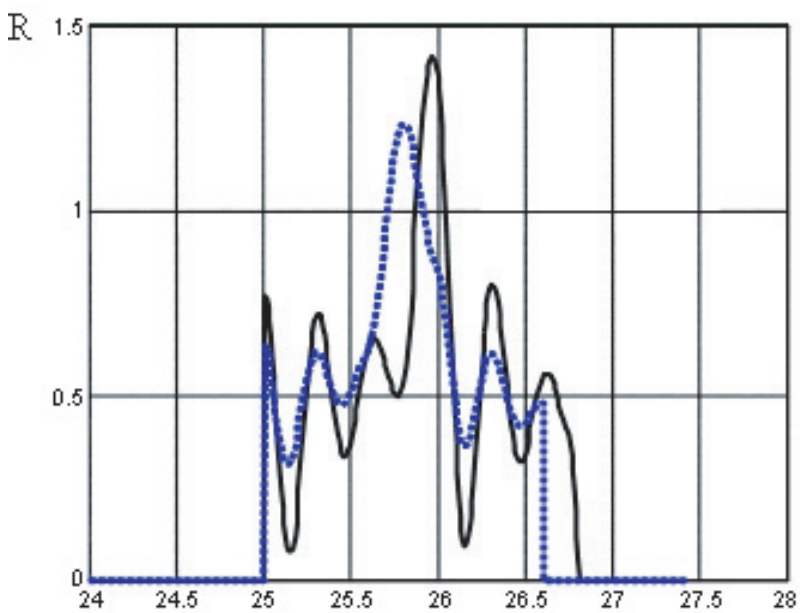

(b)

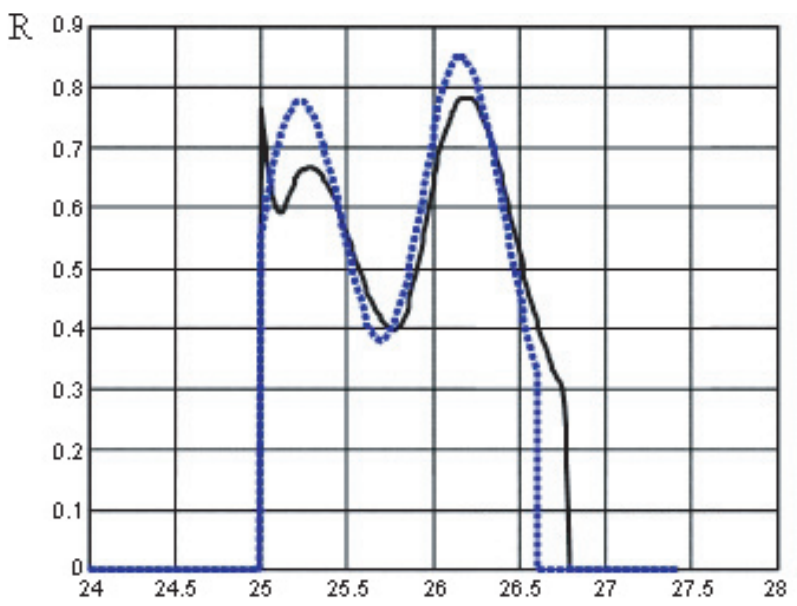

(c)

Fig. 7. Efforts sur une paire de dents en prise à différentes vitesses (facteur d'amortissement de $5 \%$ - couple de $200 \mathrm{Nm}$ ) (— Engagements prématurés, ...... Référence), (a) vitesse nominale de 1500 t.min ${ }^{-1}$, (b) vitesse nominale de 4000 t.min ${ }^{-1}$, (c) vitesse nominale de 15000 t.min ${ }^{-1}$.

\section{Conclusions}

Les résultats obtenus à l'issue de cette étude sur un modèle simplifié montrent que les contacts entre profils non conjugués au moment de l'engagement sont susceptibles d'engendrer des surcharges significatives sur les flancs de denture. Les amplitudes exactes des efforts instantanés sont difficilement quantifiables car elles semblent dépendre fortement de la loi de comportement utilisée pour décrire les efforts aux contacts en fonction des paramètres cinématiques. Il a été en particulier observé que la composante visqueuse dans l'expression des forces de contact est importante ce qui pose le problème délicat d'une modélisation précise des phénomènes dissipatifs à l'engagement. Les constats négatifs précédents relatifs aux performances dynamiques d'un engrenage droit sont, peut-être, à nuancer en ce qui concerne le comportement vibratoire global. En effet, pour des engrenages droits classiques $\left(\varepsilon_{\alpha}<2\right)$, l'augmentation du rapport de conduite sous charge tend généralement à améliorer le comportement dynamique en terme de vitesses critiques pour l'engrènement. Toutefois, d'un point de vue pratique, ces phénomènes sont indiscutablement dangereux pour la tenue des surfaces suite à l'influence croisée d'un chargement élevé et de conditions géométriques défavorables (rayons de courbure faibles à l'engagement) conduisant à des pressions extrêmement importantes.

L'ensemble des constats et remarques précédents prouve que des travaux complémentaires à cette analyse préliminaire sont indispensables. L'introduction de modèles tridimensionnels incluant les couplages entre flexion, torsion et déplacements axiaux afin de simuler le comportement d'engrenages hélicoïdaux ainsi que l'étude de l'influence de corrections de forme (hélice, profil) semblent deux des voies de développement à privilégier.

Remerciements. Les auteurs tiennent à remercier le Dr. Lars Vedmar de l'université de Lund, Suède, pour ses conseils et son aide.

\section{Références}

[1] R.W. Gregory, S.L. Harris, R.G. Munro, Dynamic behaviour of spur gears, Proc. ImechE 178 (1963) 207-226

[2] H.N. Özgüven, D.R. Houser, Mathematical models used in gear dynamics, A review, J. Sound Vib. 121 (1988) $383-411$

[3] H. Vinayak, R. Singh, Multi-body dynamics and modal analysis of compliant gear bodies, J. Sound Vib. 210 (1998) 171-214

[4] S. Baud, P. Velex, Static and dynamic tooth loading in spur and helical geared systems, Experiments and model validation, ASME J. Mech. Design 124 (2002) 334-346 
[5] A. Hösl, J. Post, F. Pfeiffer, Simulation of a Ravigneaux planetary gear of an automatic transmission, Proceedings of the International Conference on Gears, VDI, Münich, Germany, 2002, pp. 491-506

[6] R.G. Munro, N. Yildirim, D.M. Hall, Optimum profile relief and transmission error in spur gears, Proceedings of the International Conference on Gearbox Noise and Vibration, ImechE, Cambridge, 1990, pp. $35-42$

[7] C. Lee, H.H. Lin, F.B. Oswald, D.P. Townsend, Influence of linear modifications and loading conditions on the dynamic tooth load and stress of high-contactratio spur gears, ASME J. Mech. Design 113 (1991) $473-480$
[8] P. Velex, M. Maatar, A mathematical model for analyzing the influence of shape deviations and mounting errors on gear dynamics, J. Sound Vib. 191 (1996) 629-660

[9] A. Seireg, D.R. Houser, Evaluation of dynamic factors of spur and helical gears, ASME J. Eng. Ind. (1970) $504-514$

[10] T. Kasuba, J.W. Evans, An extended model for determining dynamic load in spur gearing, ASME J. Mech. Design 103 (1981) 398-408

[11] L. Vedmar, B. Henriksson, A general approach for determining dynamic forces in spur gears, ASME J. Mech. Design 120 (1998) 593-598

[12] ITI - SIM ® Simulation Software - User's manual, version 3.3, ITI GmbH, Dresden, Germany, 2001 